BMJ Open

Diabetes

Research

\& Care

\title{
Identification of candidate gene variants of monogenic diabetes using targeted panel sequencing in early onset diabetes patients
}

\author{
Dong-Hwa Lee (1) , ${ }^{1}$ Soo-Heon Kwak, ${ }^{2}$ Hee Sue Park, ${ }^{3}$ Eu Jeong Ku (D) , \\ Hyun Jeong Jeon, ${ }^{1}$ Tae Keun $\mathrm{Oh}^{1}$
}

\begin{abstract}
To cite: Lee D-H, Kwak S-H, Park HS, et al. Identification of candidate gene variants of monogenic diabetes using targeted panel sequencing in early onset diabetes patients. BMJ Open Diab Res Care 2021;9:e002217. doi:10.1136/ bmjdrc-2021-002217
\end{abstract}

- Supplemental material is published online only. To view, please visit the journal online (http://dx.doi.org/10.1136/ bmjdrc-2021-002217).

Received 14 February 2021 Accepted 1 June 2021

Check for updates

(C) Author(s) (or their employer(s)) 2021. Re-use permitted under CC BY-NC. No commercial re-use. See rights and permissions. Published by BMJ.

${ }^{1}$ Internal Medicine, Chungbuk National University Hospital, Cheongju, Korea (the Republic of)

${ }^{2}$ Internal Medicine, Seoul National University Hospital, Jongno-gu, Korea (the Republic of)

${ }^{3}$ Laboratory Medicine,

Chungbuk National University Hospital, Cheongju, Korea (the Republic of)

Correspondence to Professor Tae Keun Oh; tgohkjs@chungbuk.ac.kr

\section{ABSTRACT}

Introduction Monogenic diabetes is attributed to genetic variations in a single gene. Maturity-onset diabetes of the young (MODY) is the most common phenotype associated with monogenic diabetes, but is frequently misdiagnosed as either type 1 or type 2 diabetes. Increasing our basic understanding of genetic variations in MODY may help to improve the accuracy of providing the correct diagnosis and personalize subsequent treatment regimens in different racial populations. For this reason, this study was designed to identify nucleotide variants in early onset diabetes patients with clinically suspected MODY in a Korean population.

Research design and methods Among 2908 Korean patients diagnosed with diabetes, we selected 40 patients who were diagnosed before 30 years old and were clinically suspected of MODY. Genetic testing was performed using a targeted gene sequencing panel that included 30 known monogenic diabetes genes. The pathogenicity of the identified variants was assessed according to the American College of Medical Genetics and Genomics and Association for Molecular Pathology (ACMGAMP) guidelines.

Results A total of six rare missense variants (p.Ala544Thr in HNF1A, p.Val601lle and p.His103Tyr in ABCC8, p.Pro33Ala in PDX1, p.Gly18Glu in INS, and p.Arg164GIn in PAX4) in five distinct MODY genes were identified in five patients. In addition, a variant was identified in mitochondrial DNA at $3243 \mathrm{~A}>\mathrm{G}$ in one patient. The identified variants were either absent or detected at a rare frequency in the 1000 Genomes Project. These variants were classified as uncertain significance using the ACMGAMP guidelines.

Conclusion Using a targeted gene sequencing panel, we identified seven variants in either MODY genes or mitochondrial DNA using a Korean patient population with early onset diabetes who were clinically suspected of MODY. This genetic approach provides the ability to compare distinct populations of racial and ethnic groups to determine whether specific gene is involved in their diagnosis of MODY.

\section{INTRODUCTION}

Diabetes is a heterogeneous disease of metabolic disorders that share hyperglycemia as

\section{Significance of this study}

What is already known about this subject?

- Maturity-onset diabetes of the young (MODY) is caused by mutations in specific genes and is characterized by early onset.

- In patients with early onset diabetes, it is important to distinguish MODY from type 1 or type 2 diabetes.

- Genetic variants causing MODY have been identified using various methodologies.

What are the new findings?

- Genetic testing for 30 known monogenic diabetes genes was performed using a targeted gene sequencing panel.

- A total of six rare missense variants in five distinct MODY genes and an additional mitochondrial variant were identified, which were either absent or detected at a rare frequency in the 1000 Genomes Project.

How might these results change the focus of research or clinical practice?

- These results provide new information about genetic variants in a Korean population that could be used to help accurately predict the diagnosis of MODY.

a common clinical characteristic. Diabetes can be classified in the following three categories: (1) type 1 diabetes (T1D), (2) type 2 diabetes (T2D), (3) gestational diabetes mellitus. Although most diagnosed cases of diabetes are linked to either T1D or T2D, a considerable proportion of patients do not fit into these classifications and can fall into other causes of diabetes, which includes different forms of monogenic diabetes, including neonatal diabetes and maturityonset diabetes of the young (MODY), diseases of the exocrine pancreas, and drug-induced or chemical-induced diabetes. ${ }^{1}$

Monogenic diabetes, which is caused by variants in a single gene, accounts for $\sim 2 \%$ of all known cases of diabetes. ${ }^{2}$ The most 
common phenotype of monogenic diabetes is MODY, ${ }^{3}$ which is considered as a heterogeneous group of disorders caused by genetic variants that play a fundamentally crucial role in $\beta$-cell development, function and regulation, glucose sensing, and the proper function of the insulin gene. The prevalence of MODY is relatively rare, with only $1 \%-5 \%$ of all cases of diabetes and $1 \%-6 \%$ of diabetes in pediatric cases. ${ }^{4}$ In general, MODY is characterized by early onset (typically before 25 years of age), autosomal dominant mode of inheritance, and no dependence on insulin. ${ }^{5}$

Patients with MODY can be misdiagnosed as either T1D or T2D.${ }^{67}$ MODY can be relatively easy to distinguish from T1D because of its distinct pathogenesis, such as maintenance of $\beta$-cell function. On the other hand, the difference between MODY and T2D is more challenging due to their similar characteristics in terms of sustained insulin secretion and the existence of strong family history. However, one prominent difference between these two forms of diabetes is that MODY patients generally are not obese unlike patients with T2D. However, the degree of obesity in a specific population may confound the proper diagnosis of MODY versus T2D depending on their race or ethnicity. ${ }^{8}$ As an example, a previous study reported that approximately $10 \%-15 \%$ of Japanese children with T2D are non-obese. ${ }^{9}$ Therefore, alternate diagnostic methods were needed to complement the existing methods to properly distinguish the types of diabetes, particularly MODY, in a particular patient.

A newer method was to develop genetic testing to distinguish the various forms of diabetes in various patient populations. To date, the prevalence of specific variants in genes that cause MODY differs according to race or ethnic groups. Variants in the GCK, HNF1A, HNF4A, and $H N F 1 B$ genes are the most common causes of MODY, and they account for $32 \%, 52 \%, 10 \%$, and $6 \%$ of cases in the UK, respectively. ${ }^{10}$ In other parts of Europe, specifically France $(56 \%)$ and Italy (41\%), GCK variants were more prevalent as a suspected cause of MODY. ${ }^{11}{ }^{12}$ In Asians, there was a considerable difference in the frequencies of variants that normally cause MODY in Caucasians. In Korea, only $10 \%$ of 40 MODY or early onset T2D patients possessed known MODY gene defects, such as HNF1A (5\%), GCK (2.5\%), and HNF1B (2.5\%) among MODY 1-6 genes. ${ }^{413}$ Similar results were shown in Japan and China, only $10 \%-20 \%$ of MODY cases were caused by known MODY gene defect. ${ }^{14} 15$ This would suggest that Asians may have other as yet to be identified genetic variations that are involved in the pathogenesis of MODY.

In the present study, we designed a clinical approach to genotype patients that have been previously diagnosed as T2D to determine whether any genetic variants could be identified that would help to reclassify under the category of monogenic diabetes (or MODY). To perform this analysis, we recruited a small population of Korean patients to isolate cells for genetic testing for a target gene panel for next-generation sequencing to identify either existing or novel variants that may be associated with MODY.

\section{MATERIALS AND METHODS}

\section{Ethics statement}

This research was approved by the relevant Ethics Committees (The Institutional Review Board at Chungbuk National University Hospital, approval No. 2019-12-010001). This study was performed following the Declaration of Helsinki. ${ }^{16}$ Informed consent was obtained from all study subjects before blood sampling.

\section{Study participants}

The study participants were recruited from a hospitalbased cohort with diabetes at Chungbuk National University Hospital from March 2011. Diabetes was diagnosed clinically using the American Diabetes Association criteria. ${ }^{1}$ On the basis of onset age, especially less than 30 years old, 183 patients were enrolled in a total of 2908 patients with diabetes. Among them, patients with clinically suspected T1D $(n=101)$ who treated with insulin alone and with the presence of autoantibodies to glutamic acid decarboxylase or a fasting C-peptide level $<0.6 \mathrm{ng} / \mathrm{mL}$ were excluded. After calculation of MODY probability by the standard MODY probability calculator, ${ }^{17} 42$ patients who had less than a $50 \%$ probability of MODY were excluded. Finally, a total of 40 patients with suspected monogenic diabetes who had more than $50 \%$ of MODY probability were included in the final analysis. None of the patients had a familial relationship. A flowchart of the patient enrollment process of the study is presented in online supplemental figure 1. Clinical information, including demographics, family history, and treatment history of diabetes, physical examination, and laboratory test results for the 40 participants were obtained at the time of enrollment (table 1).

\section{Protocol for targeted panel sequencing}

In this study, DNA was extracted from peripheral blood leukocytes and was used in a targeted panel sequencing by Macrogen (Seoul, Republic of Korea). The customdesigned capture probes were previously published by Park $e a^{18}$ to include the exonic and untranslated regions of 30 genes (target region of approximately $93 \mathrm{~kb}$ ) that were known to cause either MODY, lipodystrophy, or neonatal diabetes (online supplemental table 1).

\section{Variant selection and assessment}

The sequenced reads were aligned to the human reference genome (GRCh37) using the Burrows-Wheeler Aligner (V.0.7.15). ${ }^{19}$ Using PICARD software (V.2.9.0) (http://broadinstitute.github.io/picard/) and the Genome Analysis Toolkit (V.3.8), ${ }^{20}$ PCR duplicates were removed and base quality recalibration and indel realignment were conducted. Using $\mathrm{ANNOVAR}^{21}$ and InterVar, ${ }^{22}$ annotation was conducted for all identified variants. Further annotation was performed using the Human Gene Mutation Database (HGMD) professional version release 2018.1. ${ }^{23}$

Variants selection was performed through a sequential process. At first, non-silent variants, such as 


\begin{tabular}{lc}
\hline \multicolumn{2}{l}{ Table 1 Clinical characteristics of study participants } \\
\hline Variables & Total $(\mathbf{N}=40)$ \\
\hline Male, $\mathrm{N}(\%)$ & $21(52.5)$ \\
\hline Current age, years & $25.6 \pm 5.5$ \\
\hline Age at diagnosis, years & $20.8 \pm 5.0$ \\
\hline Family history of diabetes, $\mathrm{N}(\%)$ & $32(80.0)$ \\
\hline Height, cm & $167.3 \pm 12.1$ \\
\hline Weight, kg & $77.8 \pm 22.1$ \\
\hline BMI, kg/m & $27.3 \pm 5.2$ \\
\hline SBP, $\mathrm{mm} \mathrm{Hg}$ & $127.9 \pm 17.5$ \\
HbA1c, \% & $9.4 \pm 3.0$ \\
\hline C-peptide, ng/mL & $2.0 \pm 1.1$ \\
\hline Insulin, mlU/mL & $10.8 \pm 10.5$ \\
\hline Oral antidiabetics use, N (\%) & $35(87.5)$ \\
Insulin use, N(\%) & $8(20.0)$ \\
\hline MODY probability, \% & $59.9 \pm 19.1$ \\
\hline Number of MODY criteria fulfilled & \\
\hline 1 & $2(5.0)$ \\
\hline 2 & $4(10.0)$ \\
\hline 3 & $18(45.0)$ \\
\hline 4 & $13(32.5)$ \\
\hline 5 & $3(7.5)$ \\
\hline
\end{tabular}

Data are shown as mean \pm SD or $\mathrm{N}(\%)$.

$\mathrm{BMI}$, body mass index; MODY, maturity-onset diabetes of the young; SBP, systolic blood pressure.

non-synonymous, stop gain, stop loss, start loss, frameshift, and splice site variants, were selected. Rare variants in general population with a frequency $<0.5 \%$ reported were selected. We used several population databases (Genome Aggregation Database, ${ }^{24} 1000$ Genomes Project, ${ }^{25}$ Exome Aggregation Consortium Project, ${ }^{24}$ and National Heart, Lung, and Blood Institute Exome Sequencing Project ${ }^{26}$ ) to evaluate the frequency in general population. In addition, we selected variants previously reported as being likely pathogenic or pathogenic in ClinVar or with a high confidence that the variation would cause disease in HGMD. In addition, we selected variants to have a possibility to be clinically meaningful considering the known inheritance pattern in the final analysis. The representative example of the process of variant selection is presented in online supplemental figure 2. After selecting non-silent variants, we conducted a detailed evaluation to assess the pathogenicity according to the American College of Medical Genetics and Genomics and the Association for Molecular Pathology (ACMG-AMP) guidelines. ${ }^{27}$ The ACMG-AMP guideline is consisted of two sets of criteria: (1) pathogenic or likely pathogenic variants, and (2) benign or likely benign variants. Evidence for pathogenic or likely pathogenic variants is provided as follows: one very strong (PVS1), four strong (PS1 to PS4), six moderate (PM1 to PM6), and five supporting (PP1 to PP5) attributes served as evidence of pathogenicity (online supplemental table 2). InterVar software was used to automatically determine 8 of 16 pathogenic criteria. In this study, two investigators independently analyzed the pathogenicity of each identified rare, non-silent variant according to the ACMG-AMP guidelines.

\section{RESULTS \\ Characteristics of the study participants}

A total of 40 patients with early onset diabetes were enrolled in this study, and their clinical data at the time of study enrollment are displayed in table 1 . The average age of all patients at the time of their diagnosis was $20.8 \pm 5.0$ years. A high percentage $(80 \% ; n=32)$ of the patients had a family history of diabetes, in which 10 of these 32 patients $(31.3 \%)$ had a family history of diabetes for at least three generations. The mean body mass index (BMI) of participants was $27.3 \pm 5.2 \mathrm{~kg} / \mathrm{m}^{2}$. The average HbA1c and C-peptide levels at the time of diagnosis was $9.4 \% \pm 3.0 \%$ and $2.0 \pm 1.1 \mathrm{ng} / \mathrm{mL}$, respectively. A total of 35 patients $(87.5 \%)$ were using oral antidiabetic medications, but only $8(20.0 \%)$ were using insulin. The average MODY probability was $59.9 \% \pm 19.1 \%$, but nearly half $(47.5 \% ; n=19)$ of the patients had a higher calculated MODY probability of $>75 \%$. A total of 34 patients $(85.0 \%)$ fulfilled more than three clinical diagnostic criteria of MODY, and 3 subjects $(7.5 \%)$ satisfied all five diagnostic criteria.

\section{Variant classification and prevalence of monogenic diabetes}

The average depth of coverage for each gene and percentages of the targeted region were more than $30 \mathrm{X}$ and 100X, respectively, as shown in online supplemental table 3. The average depth of coverage for the entire target region was $730 \mathrm{X}$. More than $98 \%$ of bases covered more than $30 \mathrm{X}$ in most of the genes, except for GATA4, CEL, PTF1A, KCNJ11, and GATA6. No copy number variants (CNVs) were detected in the 30 selected genes in our cohort. A total of 16 rare, non-silent variants were identified in nine distinct genes and were evaluated for pathogenicity according to the ACMG-AMP guideline. After the initial review by two study investigators who remained blinded to each other's analysis, the two investigators reached a consensus decision on the final results through a group discussion. A total of six uncertain significances and one pathogenic variant were identified (table 2). These variants were located closely in variants previously reported (figure 1). In addition to the 80 nuclear DNA variants, a pathogenic variant in mitochondrial DNA, $3243 \mathrm{~A}>\mathrm{G}$, was identified in one patient.

\section{Identified rare non-silent genetic variants of MODY}

Among the four most common MODY genes (GCK, $H N F 1 A, H N F 4 A$, and $H N F 1 B$ ), only one of these variants was identified in the present study. Other rare monogenic diabetes genes, including ABCC8, PDX1, INS-IGF2, $P A X 4$, and mitochondrial MT-TL1, were identified. The evidence for pathogenicity classification according to the ACMG-AMP guidelines is provided in table 3. The 


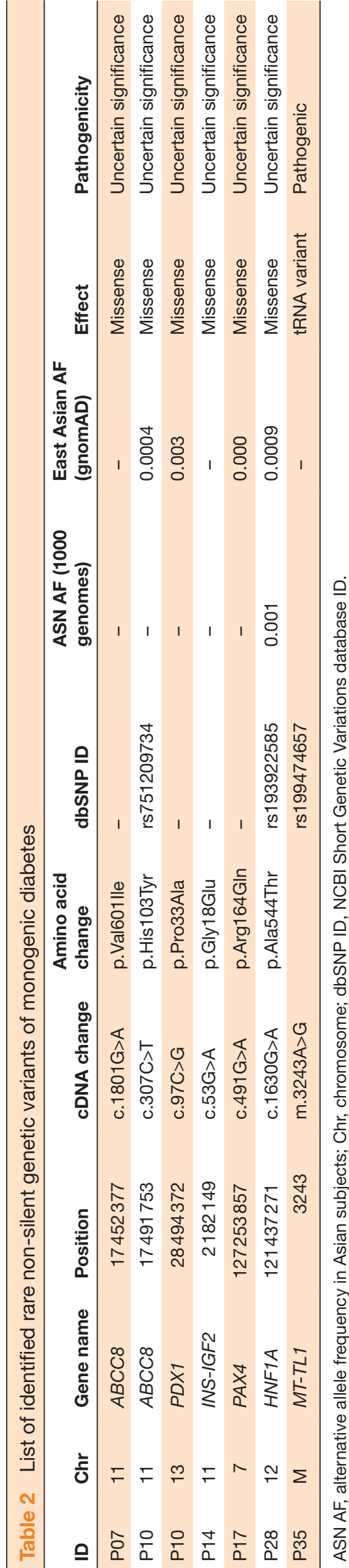

p.Ala544Thr variant of HNF1A was classified to have conflicting interpretations of pathogenicity in ClinVar. The p.Val601Ile variant of ABCC8 was classified to have uncertain significance, but it was absent from the 1000 Genomes Project. This variant was previously reported as a cause of congenital hyperinsulinism $(\mathrm{CHI}) .^{28}$ Another previously reported variant of ABCC8 gene, p.His103Tyr, which was initially categorized as likely pathogenic, ${ }^{29}$ was currently classified as uncertain significance. In the present study, p.Pro33Ala of PDX1 was identified with no report in the 1000 Genomes Project. The p.Gly18Glu of INS-IGF2 and the p.Arg164Gln of PAX4 were identified with uncertain significance, and no report in the 1000 Genomes Project. The mitochondrial variant m.3243A $>$ G, which is well known to be a causative mutation of Maternally Inherited Deafness and Diabetes (MIDD), was confirmed in five participants. Interestingly, one patient with the mitochondrial DNA 3243A $>$ G mutation had a positive maternal history of diabetes, but hearing loss was not evident in both the patient and their mother.

Clinical characteristics of patients with variants of monogenic diabetes

The variants of monogenic diabetes were identified in a total of six patients, of which two were female and four were male. The clinical characteristics of these patients are presented in table 4 . The age of the patients at the time of their diabetic diagnosis tended to be on the younger side compared with the whole population (range 18-21 years). Four out of the six patients (67\%) had a family history of diabetes, of which three out of the four patients had prolonged family history through at least three generations. The HbAlc level at diagnosis was diverse among patients (range 6.4\%-14.7\%). However, there were no patients who were treated with insulin, and $\beta$-cell function assessed by C-peptide was preserved in all patients. Eighty-three per cent of the patients (five out of six) were calculated to exhibit a MODY probability score $>75 \%$. All six patients fulfilled more than three clinical diagnostic criteria of MODY, with two of the subjects satisfied all five diagnostic criteria. There were no patients who showed other clinical phenotypes, including extrapancreatic features related to the variants.

\section{DISCUSSION}

In this study, we have investigated the genetic variants of monogenic diabetes in 40 South Korean patients with early onset diabetes using targeted panel sequencing. Among these patients, six patients had one mitochondrial and six non-silent variants in five distinct candidate genes that may be involved in monogenic diabetes, specifically MODY. All of the non-silent variants were classified to have uncertain significance using the criteria in the ACMG-AMP guidelines, but from our limited population pool, they may participate in driving the diabetic phenotype associated in MODY. 


\section{A. $\mathrm{ABCC} 8$}

c.1801G >A

c.307C $>T$

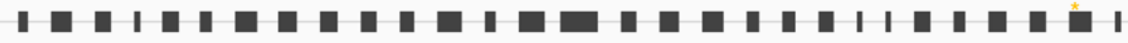

Clinvar
Pathogenic
and likely

and likely

variants (179)

c. $1817+2 \_1817+6 \mathrm{de}$

c. $1817+1 \bar{G}>T$

c. $291-2 A>G$

c. $1792 C>T$

c. $1793 \mathrm{G}>\mathrm{A}$

B. PDX1

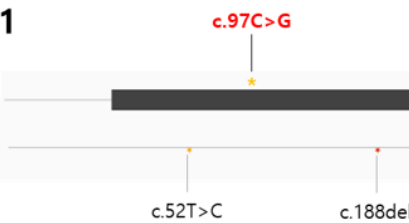

C. INS-IGF2

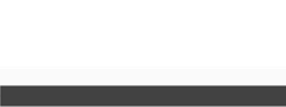

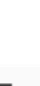

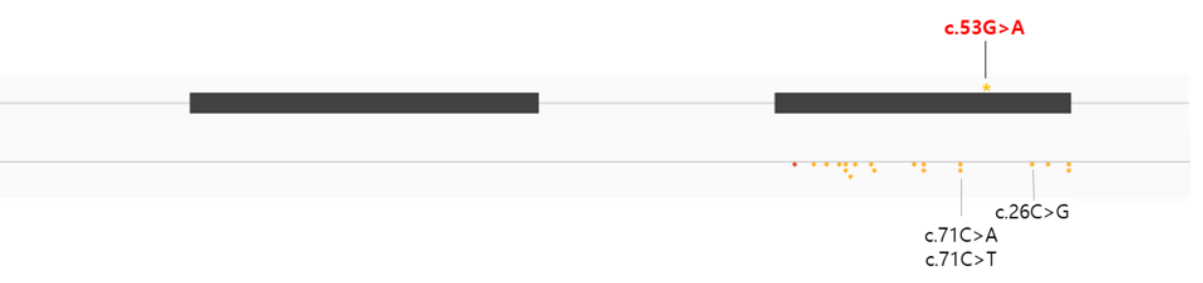

D. PAX4

Clinvar
Pathogenic

Pathogenic
and likely

pathogenic

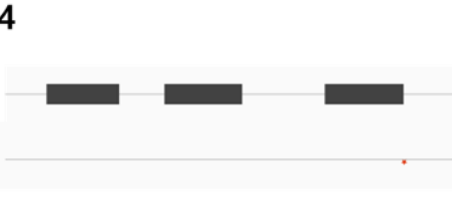

\section{E. HNF1A}
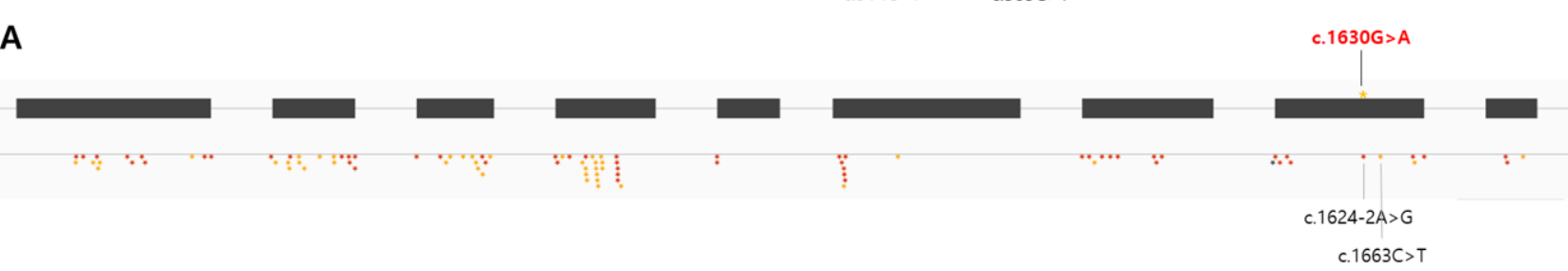

Figure 1 The spectrum of genetic variants in five MODY genes identified. Each note above represents a variant in this study. Each colored asterisk below represents a genetic mutation reported in ClinVar (red-putative loss off function, orangemissense, black - other). (A) ABCC8 gene. (B) PDX1 gene. (C) INS-IGF2 gene. (D) PAX4 gene. (E) HNF1A gene. Adapted from gnomAD (https://gnomad.broadinstitute.org/).

Although MODY accounts for a small percentage $(1 \%-2 \%)$ of all cases of diabetes, the accurate diagnosis of MODY is very important for patients and their families to ensure that the proper treatment regimen is initiated to treat their malady. Once we accumulate sufficient genetic data in patients with diabetes, genetic testing and counseling will likely have a major impact in a positive way to provide more accurate and earlier diagnosis of diabetes, which will inevitably improve the long-term outcome of the affected patient through optimized treatment protocols. In fact, there is emerging evidence that genetic testing has shown success in distinguishing monogenic diabetes subtypes, MODY, and neonatal diabetes. ${ }^{30}$ In particular, MODY can be diagnosed by direct sequencing

\begin{tabular}{|c|c|c|c|c|c|c|c|c|c|c|c|c|c|c|c|}
\hline Gene & Variant & PVS1 & PS1-5 & PM1 & PM2 & PM3 & PM4 & PM5 & PM6 & PP1 & PP2 & PP3 & PP4 & PP5 & Final \\
\hline$A B C C 8$ & c. $1801 \mathrm{G}>\mathrm{A}$ & & & + & + & & & & & & & + & & & US \\
\hline$A B C C 8$ & c. $307 \mathrm{C}>\mathrm{T}$ & & & & + & & & & & & & + & & & US \\
\hline$P D X 1$ & c. $.97 \mathrm{C}>\mathrm{G}$ & & & & + & & & + & & & & + & & & US \\
\hline INS-IGF2 & c. $53 \mathrm{G}>\mathrm{A}$ & & & + & + & & & & & & + & & & & US \\
\hline PAX4 & c. $491 \mathrm{G}>\mathrm{A}$ & & & + & + & & & & & & & + & & & US \\
\hline HNF1A & c. $1630 \mathrm{G}>\mathrm{A}$ & & & & + & & & & & & & & & + & US \\
\hline
\end{tabular}

US, uncertain significance. 


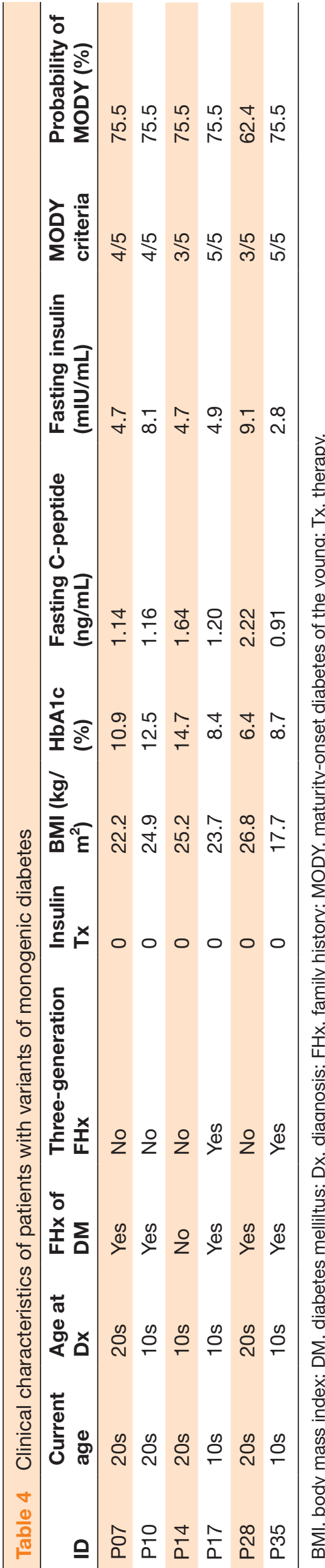

with up to $100 \%$ sensitivity. ${ }^{10}$ The major drawback with genetic testing is the relatively high cost due in part to its availability in select specialized laboratories. Therefore, it is important to use the patient history and clinical values to select patients with clinically suspected monogenic diabetes. In our study using a small pilot group of preselected Korean patients with suspected probability of MODY, we performed a genetic analysis using a targeted gene panel.

Overall, variations in HNF1A are considered to be the most common cause of MODY in Europe, North America and Asia, ${ }^{31}$ with a current total of 414 different HNF1A variants out of 1247 families. ${ }^{32}$ In Asians, the prevalence of HNF1A variations was initially detected in 5\%-8\% of patients diagnosed with MODY. More specifically, in Japanese and Chinese, the prevalence of HNF1A variants was $8 \%$ and $5 \%$, respectively. ${ }^{33}{ }^{34}$ In Korea, one patient (6.3\%) with an HNF1A R267L mutation was detected among 16 unrelated patients diagnosed with T2D before the age of 35 years and a family history of autosomal dominant inheritance of T2D for at least two generations. ${ }^{35}$ In this current study, the prevalence in the detection of an HNF1A variant was lower $(2.5 \%)$ compared with the other studies, but this was consistent with another recent study by our group using a similar selection criteria and sequencing protocol. ${ }^{18}$ In our other study, we found 3 out of 109 patients having one of three distinct HNF1A variants (p.Tyr166Asn, p.Leu26Gln, p.Val567Ile), which are classified as likely pathogenic. ${ }^{18}$ The present study detected a distinct HNF1A variant (p.Ala544Thr) that was not identified in our other study, and this particular variant is listed in the 1000 Genomes Project with an as yet to be determined biological role due to conflicting interpretations of its pathogenicity in ClinVar.

In our study, the patient with the HNF1A variant did not have a family history, and his BMI was $>25 \mathrm{~kg} / \mathrm{m}^{2}$. The patient exhibited good glycemic control using his treatment with metformin and DPP4 inhibitors, where his latest $\mathrm{HbA1c}$ level was $6.3 \%$. Generally, patients with HNF1A mutations are sensitive to sulfonylurea. Considering the clinical characteristics of this patient and other previous reports, it still remains unclear whether this distinct HNF1A variant influenced the onset of MODY. No other variants in other common MODY genes, including $G C K, H N F 4 A, H N F 1 B$, were identified in this patient. Overall, the relatively lower prevalence of the HNF1A variants within Asian countries, particularly our recent findings in Korea, demonstrates the importance of genetic testing and analysis between distinct racial and ethnic groups, but also may be attributed to the criteria used to select patients. ${ }^{36}$

Novel variants in other genes, including $A B C C 8, P D X 1$, $I N S$, and PAX4, believed to be involved in the pathogenesis of MODY, although on a rarer basis were identified in our study. The p.Val601Ile and p.His103Tyr in ABCC were discovered in two patients. In a previous study conducted in the UK, p.Val601Ile was identified in patients with $\mathrm{CHI}^{28}$ Furthermore, it was reported that dominant 
inheritance of ABCC8 mutations can cause CHI with predisposition to insulin deficiency and diabetes later in life. ${ }^{37}$ The p.His103Tyr variant was reported as likely pathogenic in a Korean patient with diabetes. ${ }^{29}$ The clinical manifestations of MODY caused by $A B C C 8$ gene variations are similar to those with HNF4A/1A mutations. ${ }^{4}$ The two patients in this study did not show insulin deficiency or a history that would suggest CHI. In addition, they were treated with three classes of oral glycemic agents. Their clinical symptoms showed inconsistencies with known features associated with MODY due to the ABBCC8 mutation.

In the PDX1 gene, a novel variant p.Pro33Ala was identified in this study, which was not listed in the 1000 Genomes Project. A previous study demonstrated that a missense variant changing the amino acid from proline to threonine (PDX1 ${ }^{\mathrm{P} 33 \mathrm{~T} / \mathrm{P} 33 \mathrm{~T}}$ ) led to a deterioration of $\beta$-cell development and function. ${ }^{38}$ The p.Gly18Glu in INS gene and the p.Arg164Gln in PAX4 gene were also novel variants that were not previously reported. The patients with these variants did not have typical clinical features, such as ketosis, or requirement of insulin. Little is known about these variants due to their apparent rarity, so further research is needed to better understand whether these variants play a role in the pathogenesis of MODY.

In one patient with the $\mathrm{A}>\mathrm{G}$ transition at position 3243 in the mitochondrial tRNALeu-encoding (UUR) gene (m.3243A $>\mathrm{G}$, MT-TL1), this variant is believed to be the most common mutation causing MIDD. ${ }^{39}$ This syndrome usually affects metabolically active organs (such as endocrine pancreas and cochlea) and is accompanied by the severe childhood neurological phenotype mitochondrial encephalomyopathy, lactic acidosis, and strokelike episodes (MELAS) ${ }^{40}$ This one patient presented a strong maternal inheritance feature where the patient's maternal grandmother and mother were diagnosed with diabetes. However, the patient was not deaf, and there were no other features associated with MELAS. It is known that the clinical manifestation associated with MIDD and MELAS displays heterogeneous phenotypes, ${ }^{40}$ so it is possible that our patient might have only a mild form of MIDD.

As with most association studies, there are limitations to the interpretation of our findings. First, there are several useful tools that have been developed to screen for MODY, including the use of clinical criteria and MODY probability calculators. In general, the criteria include clinical characteristics, such as age of onset for diabetes, BMI, family history, pancreatic function, and insulin use. However, no consensus clinical criteria have been used in all published studies. Moreover, the MODY probability calculator consists of eight clinical factors, ${ }^{17}$ which has been developed using clinical information acquired mostly from Europeans and has not been validated in other races. In our results, our patient population with the identified variants fulfilled more than three of five clinical criteria and exhibited a high MODY probability score. Therefore, these tools may not be limited in our study and could be properly applied to select the patients suspected of MODY before genetic testing. Second, the number of patients was relatively small. Because the inclusion criteria of the patients with age at diagnosis was limited under 30 years, there is a possibility of missing patients with monogenic diabetes who were diagnosed after 30 years old. However, the molecular genetic diagnosis rate in patients with an onset age $>40$ years was reported to be only $0.6 \%{ }^{41}$ Third, the variants identified in this study were classified as uncertain significance except for one variant found in the mitochondria. These might be attributed to the lack of functional studies to identify variants and the absence of sequencing within family members. Lastly, many of the patients did not have variants for MODY, even though there were clinical data that suspected their genetic predisposition. It is unclear whether the unidentified variants for MODY, so-called MODYX, might exist or not in these patients. In addition, the present analysis did not include promoter variants so it may be possible that variants existed outside of the coding region.

In conclusion, our present study used targeted panel sequencing to identify seven variants, of which five were novel, in six distinct patients among a population of 40 patients with early onset diabetes and were clinically suspected of MODY. The results from this study continue to accumulate more data demonstrating the potential benefit of target panel sequencing to identify existing and new variants in genes associated with MODY for future development of genetic biomarkers for generelated diabetes, especially in distinct racial and ethnic populations.

Contributors TKO and D-HL designed the study. D-HL researched the literature, interpreted the data, and wrote the manuscript and figures. EJK and HJJ contributed to data collection. HJJ and S-HK reviewed the manuscript and contributed to the discussion. All authors critically reviewed the manuscript and approved its submission. TKO is the guarantor of this work and, as such, had full access to all of the data in the study and take responsibility for the integrity of the data and the accuracy of the data analysis.

Funding This study was supported by a grant from the Korean Endocrine Society in 2020. The funding sources were not involved in oversight or design of the study, in the analysis or interpretation of the data, or in the decision to submit the manuscript for publication.

Competing interests None declared.

Patient consent for publication Not required.

Provenance and peer review Not commissioned; externally peer reviewed.

Data availability statement Data are available on reasonable request. All data relevant to the study are included in the article or uploaded as supplemental information. If there are requests for data sharing, contact to corresponding author by email (tgohkjs@chungbuk.ac.kr).

Supplemental material This content has been supplied by the author(s). It has not been vetted by BMJ Publishing Group Limited (BMJ) and may not have been peer-reviewed. Any opinions or recommendations discussed are solely those of the author(s) and are not endorsed by BMJ. BMJ disclaims all liability and responsibility arising from any reliance placed on the content. Where the content includes any translated material, BMJ does not warrant the accuracy and reliability of the translations (including but not limited to local regulations, clinical guidelines, terminology, drug names and drug dosages), and is not responsible for any error and/or omissions arising from translation and adaptation or otherwise. 
Open access This is an open access article distributed in accordance with the Creative Commons Attribution Non Commercial (CC BY-NC 4.0) license, which permits others to distribute, remix, adapt, build upon this work non-commercially, and license their derivative works on different terms, provided the original work is properly cited, appropriate credit is given, any changes made indicated, and the use is non-commercial. See: http://creativecommons.org/licenses/by-nc/4.0/.

ORCID iDs

Dong-Hwa Lee http://orcid.org/0000-0002-1552-3205

Eu Jeong Ku http://orcid.org/0000-0001-5533-4989

\section{REFERENCES}

1 American Diabetes Association. 2. Classification and Diagnosis of Diabetes: Standards of Medical Care in Diabetes-2020. Diabetes Care 2020;43:S14-31.

2 Murphy R, Ellard S, Hattersley AT. Clinical implications of a molecular genetic classification of monogenic beta-cell diabetes. Nat Clin Pract Endocrinol Metab 2008;4:200-13.

3 Shepherd M, Shields B, Hammersley S, et al. Systematic Population Screening, Using Biomarkers and Genetic Testing, Identifies 2.5\% of the U.K. Pediatric Diabetes Population With Monogenic Diabetes. Diabetes Care 2016;39:1879-88.

$4 \mathrm{Kim} \mathrm{SH}$. Maturity-Onset diabetes of the young: what do clinicians need to know? Diabetes Metab J 2015;39:468-77.

5 Fajans SS, Bell GI, Polonsky KS. Molecular mechanisms and clinical pathophysiology of maturity-onset diabetes of the young. $N$ Engl $J$ Med 2001;345:971-80.

6 Hattersley AT, Greeley SAW, Polak M, et al. ISPAD clinical practice consensus guidelines 2018: the diagnosis and management of monogenic diabetes in children and adolescents. Pediatr Diabetes 2018;19 Suppl 27:47-63.

7 Thanabalasingham G, Pal A, Selwood MP, et al. Systematic assessment of etiology in adults with a clinical diagnosis of young-onset type 2 diabetes is a successful strategy for identifying maturity-onset diabetes of the young. Diabetes Care 2012;35:1206-12

8 Ogden CL, Carroll MD, Lawman HG, et al. Trends in obesity prevalence among children and adolescents in the United States, 1988-1994 through 2013-2014. JAMA 2016;315:2292-9.

9 Urakami T, Miyata M, Yoshida K, et al. Changes in annual incidence of school children with type 2 diabetes in the Tokyo metropolitan area during 1975-2015. Pediatr Diabetes 2018;19:1385-92.

10 Kavvoura FK, Owen KR. Maturity onset diabetes of the young: clinical characteristics, diagnosis and management. Pediatr Endocrinol Rev 2012;10:234-42.

11 Malecki MT, Jhala US, Antonellis A, et al. Mutations in NeuroD1 are associated with the development of type 2 diabetes mellitus. Nat Genet 1999;23:323-8.

12 Massa O, Meschi F, Cuesta-Munoz A, et al. High prevalence of glucokinase mutations in Italian children with MODY. Influence on glucose tolerance, first-phase insulin response, insulin sensitivity and BMI. Diabetologia 2001;44:898-905.

13 Hwang JS, Shin $\mathrm{CH}$, Yang SW, et al. Genetic and clinical characteristics of Korean maturity-onset diabetes of the young (MODY) patients. Diabetes Res Clin Pract 2006;74:75-81.

14 Nishigori H, Yamada S, Kohama T, et al. Mutations in the hepatocyte nuclear factor-1 alpha gene (MODY3) are not a major cause of earlyonset non-insulin-dependent (type 2) diabetes mellitus in Japanese. $J$ Hum Genet 1998;43:107-10.

15 Xu JY, Dan QH, Chan V, et al. Genetic and clinical characteristics of maturity-onset diabetes of the young in Chinese patients. Eur $\mathrm{J}$ Hum Genet 2005;13:422-7.

16 World Medical Association. World Medical association Declaration of Helsinki: ethical principles for medical research involving human subjects. JAMA 2013;310:2191-4.

17 Shields BM, McDonald TJ, Ellard S, et al. The development and validation of a clinical prediction model to determine the probability of MODY in patients with young-onset diabetes. Diabetologia 2012;55:1265-72.

18 Park SS, Jang SS, Ahn CH. Identifying pathogenic variants of monogenic diabetes using targeted panel sequencing in an East Asian population. J Clin Endocrinol Metab 2019.
19 Li H, Durbin R. Fast and accurate short read alignment with Burrows-Wheeler transform. Bioinformatics 2009;25:1754-60.

20 McKenna A, Hanna M, Banks E, et al. The genome analysis toolkit: a MapReduce framework for analyzing next-generation DNA sequencing data. Genome Res 2010;20:1297-303.

21 Wang K, Li M, Hakonarson H. ANNOVAR: functional annotation of genetic variants from high-throughput sequencing data. Nucleic Acids Res 2010;38:e164.

$22 \mathrm{Li} \mathrm{Q}$, Wang K. InterVar: clinical interpretation of genetic variants by the 2015 ACMG-AMP guidelines. Am J Hum Genet 2017;100:267-80.

23 Stenson PD, Ball EV, Mort M, et al. The human gene mutation database (HGMD) and its exploitation in the fields of personalized genomics and molecular evolution. Curr Protoc Bioinformatics 2012; Chapter 1:Unit1.13.

24 Lek M, Karczewski KJ, Minikel EV, et al. Analysis of protein-coding genetic variation in 60,706 humans. Nature 2016;536:285-91.

251000 Genomes Project Consortium, Auton A, Brooks LD, et al. A global reference for human genetic variation. Nature 2015;526:68-74.

26 Fu W, O'Connor TD, Jun G, et al. Analysis of 6,515 exomes reveals the recent origin of most human protein-coding variants. Nature 2013;493:216-20.

27 Richards S, Aziz N, Bale S, et al. Standards and guidelines for the interpretation of sequence variants: a joint consensus recommendation of the American College of medical genetics and genomics and the association for molecular pathology. Genet Med 2015;17:405-23.

28 Arya VB, Guemes M, Nessa A, et al. Clinical and histological heterogeneity of congenital hyperinsulinism due to paternally inherited heterozygous ABCC8/KCNJ11 mutations. Eur J Endocrinol 2014;171:685-95.

29 Kwak SH, Jung C-H, Ahn CH, et al. Clinical whole exome sequencing in early onset diabetes patients. Diabetes Res Clin Pract 2016;122:71-7.

30 Hattersley AT, Patel KA. Precision diabetes: learning from monogenic diabetes. Diabetologia 2017;60:769-77.

31 Hattersley AT. Maturity-onset diabetes of the young: clinical heterogeneity explained by genetic heterogeneity. Diabet Med 1998;15:15-24.

32 Colclough K, Bellanne-Chantelot C, Saint-Martin C, et al. Mutations in the genes encoding the transcription factors hepatocyte nuclear factor 1 alpha and 4 alpha in maturity-onset diabetes of the young and hyperinsulinemic hypoglycemia. Hum Mutat 2013;34:669-85.

33 Tonooka N, Tomura $\mathrm{H}$, Takahashi $\mathrm{Y}$, et al. High frequency of mutations in the HNF-1alpha gene in non-obese patients with diabetes of youth in Japanese and identification of a case of digenic inheritance. Diabetologia 2002;45:1709-12.

$34 \mathrm{Ng} \mathrm{MC}$, Cockburn BN, Lindner TH, et al. Molecular genetics of diabetes mellitus in Chinese subjects: identification of mutations in glucokinase and hepatocyte nuclear factor-1alpha genes in patients with early-onset type 2 diabetes mellitus/MODY. Diabet Med 1999:16:956-63.

35 Kim K-A, Kang K, Chi Y-I, et al. Identification and functional characterization of a novel mutation of hepatocyte nuclear factor-1alpha gene in a Korean family with MODY3. Diabetologia 2003;46:721-7.

36 Misra S, Shields B, Colclough K, et al. South Asian individuals with diabetes who are referred for MODY testing in the UK have a lower mutation pick-up rate than white European people. Diabetologia 2016;59:2262-5.

37 Bowman P, Flanagan SE, Edghill EL, et al. Heterozygous ABCC8 mutations are a cause of MODY. Diabetologia 2012;55:123-7.

38 Wang X, Sterr M, et al. Point mutations in the Pdx1 transactivation domain impair human $\beta$-cell development and function. Mol Metab 2019;24:80-97.

39 Davis RL, Sue CM. The genetics of mitochondrial disease. Semin Neurol 2011;31:519-30.

40 Murphy R, Turnbull DM, Walker M, et al. Clinical features, diagnosis and management of maternally inherited diabetes and deafness (MIDD) associated with the $3243 \mathrm{~A}>\mathrm{G}$ mitochondrial point mutation. Diabet Med 2008;25:383-99.

41 Bansal V, Gassenhuber J, Phillips T, et al. Spectrum of mutations in monogenic diabetes genes identified from high-throughput DNA sequencing of 6888 individuals. BMC Med 2017;15:213. 